\title{
El teorema del Trabajo y la Energía en marcos de referencias rotacionales
}

\author{
Work - Energy theorem in rotational reference frames \\ D.M.Fernández ${ }^{* 1}$, M.F.Carusela ${ }^{1}$, C.D.El Hasi ${ }^{1}$, F. Lopez Arriazu ${ }^{2}$ \\ ${ }^{1}$ Instituto de Ciencias, Universidad Nacional de General Sarmiento, J. M Gutierrez 1150, Los Polvorines, Argentina. \\ ${ }^{2}$ Facultad de Ciencias Exactas y Naturales, Universidad de Buenos Aires, Buenos Aires, Argentina.
}

Recebido em 17 de março de 2016. Aceito em 28 de março de 2016

\begin{abstract}
En los libros de texto estándar de física universitaria, el Teorema del Trabajo y la Energía se presenta generalmente para sistemas de referencia inerciales, en los cuales está claro que la energía cinética se conserva cuando no hay trabajo neto de las fuerzas de interacción ¿Pero qué sucede cuando la energía y el trabajo se calculan en un marco de referencia no inercial? En general, en los textos básicos de física esta importante cuestión no es tratada. En este trabajo buscamos contribuir, en este aspecto, con una propuesta de actividades que permitan presentar y discutir este teorema parados como observadores en rotación relativa, haciendo hincapié explícitamente en las diferencias entre una y otra descripción. Esta propuesta tiene como objetivo la presentación gradual, mediante la discusión, de los conceptos a partir de ejemplos sencillos y prácticos, pero utilizando nociones básicas del formalismo vectorial.

Palavras-chave: Trabajo y energía, Sistemas de referencia no inerciales, Física educativa.
\end{abstract}

In standard textbooks of college physics, Theorem of Work and Energy is generally presented for inertial reference systems in which it is clear that the kinetic energy is conserved when there is not net work of the forces of interaction. But what happens when energy and work are calculated on a non-inertial reference frame? In general, in basic physics textbooks this important issue is not treated. In this paper we seek to contribute, in this respect, with a proposal of activities to present and discuss this theorem standing for observers in relative rotation, explicitly emphasizing the differences between each description. This proposal aims at a gradual introduction, by discussion of concepts from simple and practical examples, but using the basic knowledge of vector formalism.

Keywords: Work and Energy, Non-inertial reference frame, Physics Education.

\section{Introducción}

El concepto de energía es de los más fundamentales de las Ciencias y su validez y aplicabilidad se extiende a todas las ramas de la Física. Particularmente, el Teorema del Trabajo y la Energía (TTE), que establece que el trabajo hecho por la fuerza neta aplicada sobre una partícula provoca un cambio en su energía cinética, juega un rol muy importante en Mecánica. Recordemos, no obstante, que éste no es un principio básico o general, sino que está basado en la definición de trabajo y en la segunda ley de Newton.

*Endereço de correspondência: dfernand@ungs.edu.ar
La presentación del concepto de energía y de su conservación en cursos introductorios de física presenta dificultades, aun cuando diversas propuestas han sido dadas para enfrentar el problema [5]. Asimismo, se pueden dar varias definiciones de trabajo como las que se pueden señalar en [6], [5]. Por otra parte, cuando se trata de un sistema de varias partículas es necesario tener en cuenta que la diferencia entre el trabajo hecho sobre el centro de masa y el trabajo hecho sobre las partículas se halla esencialmente ligada a los cambios en las posiciones relativas de las partículas. Esta energía interna debe ser incluida en la expresión general.

Por otro lado, resulta sumamente relevante la comparación de las leyes de Newton hechas por dos 
observadores en movimiento relativo. Esta comparación conduce al principio de relatividad galileano. La invariancia de las leyes de Newton es ampliamente analizada en la mayoría de los libros de texto de física elemental, pero usualmente el balance de trabajo y energía está descripto desde un dado marco de referencia sin analizarse la invariancia galileana. Esto ocurre porque no siempre es simple extender las leyes físicas desde un observador a otro en movimiento relativo. Pero en el caso del TTE, al no ser un principio básico, resulta muy importante e ilustrativo verificar su validez.

Para un movimiento traslacional no uniforme se muestra la diferencia de energía cinética $\mathrm{K}$ y trabajo mecánico $\mathrm{W}$ entre observadores inerciales y no inerciales [2], donde se obtiene una expresión para el trabajo hecho por fuerzas inerciales $\mathrm{W}_{\text {inercial }}$ para el caso de un sistema de partículas. Por otra parte, bajo la suposición de un movimiento relativo traslacional uniforme se ha establecido una profunda relación entre el TTE y el teorema del impulso [1].

Generalmente, los resultados obtenidos para movimiento relativo traslacional no son simplemente aplicados a sistemas rotantes, como puede ser visto en muchos libros de texto ([3], [4]) donde la dinámica rotacional sólo es tratada después de un cuidadoso estudio de la mecánica traslacional. No obstante, esta no es una cuestión trivial que serviría sólo para analizar la física sobre una calesita, por ejemplo. La Tierra, sobre la que efectuamos la mayoría de nuestros experimentos en Física, es efectivamente un sistema rotante y los efectos de esta rotación deben ser tenidos en cuenta, por ejemplo al analizar la dinámica atmosférica.

El tema se enseña a los alumnos después de haber visto cinemática, dinámica (de uno y varios cuerpos) y trabajo y energía (conservación de la energía). Los contenidos mencionados se tratan habitualmente dentro del marco de los sistemas inerciales. Todos estos temas luego se retoman y encuadran en lo que corresponde a sistemas no inerciales (SIN) (primero en traslación y luego en rotación).

Es decir, la propuesta desarrollada en este trabajo puede pensarse como una instancia integradora de los temas antes señalados. Además, podría ser retomada en cursos más avanzados, como ser una Mecánica Lagrangiana.

"El Teorema del Trabajo y la Energía y los sistemas de referencias rotacionales" podría considerarse como central para el estudio de la mecánica ya que la Tierra, el sistema donde hemos construido todo el edificio de la Física, es un sistema rotante (además de estar en traslación). Al establecer las leyes de la Física, se asume implícitamente que se formulan desde algún sistema en "reposo", el referencial absoluto de Newton o el asociado a las estrellas fijas de Mach. Es muy importante tener presente cuáles son las hipótesis que se realizan para la descripción de un fenómeno. En particular, para establecer claramente los límites de validez de las leyes Físicas. Y en general, cuando se explican los temas troncales de dinámica-energía en los libros de Física I no se hace ninguna aclaración sobre los sistemas donde tienen validez, hasta ingresar específicamente al tema de SNI. Y aún en este caso no se aborda en general el tema de trabajo-energía.

La meta ${ }^{1}$ de presentar este tipo de problema a los alumnos es que éstos logren revisar críticamente lo aprendido con anterioridad, para poder apreciar los límites de validez de la descripción de un problema y del Teorema de Conservación de la Energía. Los alumnos deberían ser capaces de establecer la relación entre las formas de describir la física de dos observadores en movimiento rotacional relativo.

En el presente artículo mostraremos la extensión del TTE en sistemas de referencias no inerciales rotacionales presentando algunos ejemplos ilustrativos $[13,14]$. En la Sección 2. presentamos explícitamente el trabajo hecho por las fuerzas inerciales y discutimos las diferentes interpretaciones dadas tanto para observadores inerciales como no inerciales. La Sección 3. contiene algunos problemas para ilustrar nuestro enfoque. En la Sección 4. presentamos las conclusiones de nuestro trabajo.

\section{Formulación del Problema}

Proponemos aquí un enfoque que hecha luz sobre el origen de la diferencia de energía medida por dos observadores en movimiento relativo, en conexión con el trabajo hecho por las fuerzas de interacción y por las fuerzas inerciales, discusión que usualmente no es tenida en cuenta en la mayoría de los libros de texto.

Supongamos dos marcos de referencia, uno en reposo (o inercial) $\sum$ y el otro rotacional (o no inercial) $\sum$ ' girando con velocidad angular $\omega$ relativa a $\sum$. Una partícula de masa $m$ cuya posición en

\footnotetext{
${ }^{1}$ Entiéndase por "meta" al logro básico de comprensión al que apuntan docentes y alumnos [9].
} 
$\sum$ sea $\mathbf{r}$ tendrá en $\sum$ ' una posición $\mathbf{r}$ '. Como sus orígenes de coordenadas coinciden, la posición de la partícula es la misma en ambos sistemas, pero serán expresadas de diferentes formas: $\mathbf{r}^{\prime}=\mathbf{M r}$ donde $\mathbf{M}$ representa la matriz de rotación (ver apéndice).

Sea una fuerza neta $\mathbf{F}$ actuando sobre la partícula, entonces desde el punto de vista de un observador en $\sum$, el TTE se expresa como: $m \mathbf{v} \cdot d \mathbf{v}=\mathbf{F} \cdot d \mathbf{r}$ ó $d K=$ $d W$. El observador en $\sum$ ' ve la misma fuerza, pero él debe incluir las llamadas fuerzas inerciales (fuerza de Coriolis y fuerza centrífuga) para preservar la validez de la segunda ley de Newton:

$$
\begin{aligned}
& m \frac{d \mathbf{v}^{\prime}}{d t}=\mathbf{F}-m \\
& \times\left[\frac{d \boldsymbol{\omega}}{d t} \times \mathbf{r}^{\prime}+2 \boldsymbol{\omega} \times \mathbf{v}^{\prime}+\boldsymbol{\omega} \times \boldsymbol{\omega} \times \mathbf{r}^{\prime}\right] .
\end{aligned}
$$

Aplicando la definición usual de trabajo en marcos de referencias rotantes el TTE todavía se sostiene y expresa como $[1,10]$ :

$$
\begin{aligned}
& m d \mathbf{v}^{\prime} \cdot \mathbf{v}^{\prime}=\left[\mathbf{F}-m \frac{d \boldsymbol{\omega}}{d t} \times \mathbf{r}^{\prime}-2 m \boldsymbol{\omega} \times \mathbf{v}^{\prime}\right. \\
& \left.-m\left(\boldsymbol{\omega} \times \boldsymbol{\omega} \times \mathbf{r}^{\prime}\right)\right] \cdot d \mathbf{r}^{\prime} .
\end{aligned}
$$

En forma abreviada: $d K^{\prime}=d W^{\prime}$.

Es posible relacionar las cantidades medidas en ambos sistemas y ver que el trabajo medido en $\sum$ ' es igual al trabajo hecho por las fuerzas de interacción (como las medidas en $\sum$ ) más un término debido a las fuerzas inerciales:

$$
d W^{\prime}=d W+d W_{\text {rot }}
$$

Mientras que lo mismo sucede para la energía cinética:

$$
d K^{\prime}=d K+d W_{r o t}
$$

Donde el trabajo rotacional es expresado por (ver apéndice):

$$
\begin{aligned}
& d W_{\text {rot }}=-(\mathbf{r} \times \mathbf{F}) \cdot d \boldsymbol{\theta}-m(\boldsymbol{\omega} \times \boldsymbol{\omega} \times \mathbf{r}) \cdot d \mathbf{r} \\
& -m\left(\mathbf{r} \times \frac{d \mathbf{r}}{d t}\right) \cdot d \boldsymbol{\omega}
\end{aligned}
$$

Podemos ver que el primer término se debe al trabajo hecho por el momento de las fuerzas aplicadas, el segundo corresponde al trabajo hecho por la fuerza centrífuga como una función de las variables fijas del sistema. Mientras tanto, el tercer término está relacionado a la variación de la velocidad angular.

\section{Problemas}

En este apartado presentamos algunos problemas (o desempeños para los alumnos) que pueden agruparse en tres niveles de complejidad. El problema 1 tiene carácter exploratorio y podría ser resuelto por los propios alumnos con los conocimientos adquiridos previamente. Los problemas $\mathbf{2}$ y $\mathbf{3}$ son propios del curso y se espera que los alumnos puedan resolverlos con la asistencia del docente. El problema 4 involucra una síntesis de los conceptos aprendidos [9].

Estos problemas muestran explícitamente cómo los diferentes términos inerciales afectan la expresión de la energía tal como lo ven los dos observadores en movimiento relativo rotacional. Con el fin de obtener una visión un poco más profunda de los conceptos involucrados pero sin un desarrollo tedioso de cálculos, consideraremos un sistema en rotación con velocidad angular constante $\boldsymbol{\omega}=\omega \leftrightharpoons z, \mathrm{y}$ asumiremos que el origen de coordenadas de ambos sistemas coinciden, por lo que los ejes $\mathbf{z}$ y z' son paralelos en todo momento (Ver figura 1).

En los próximos problemas usaremos la ecuación (5), donde el último término es cero bajo la suposición de que la velocidad angular es constante.

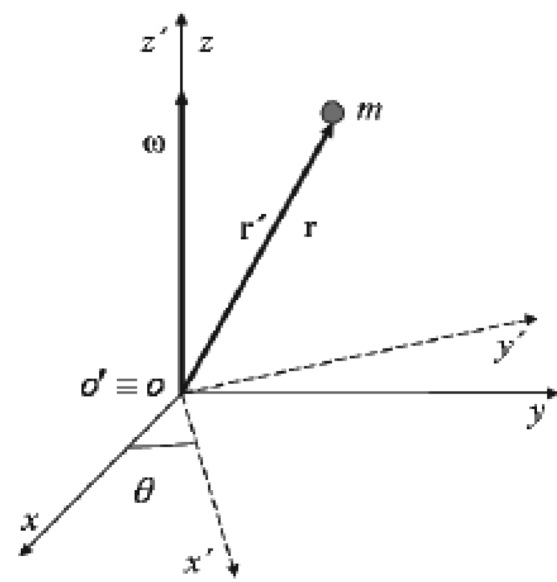

Figura 1: Dos sistemas en movimiento relativo rotacional 


\subsection{Problema 1}

Consideramos una partícula de masa $m$ fija a un disco rotante de masa despreciable. Supongamos que la partícula se sostiene en su posición debido a la fuerza de fricción estática $\mathbf{F}$. De la aplicación de la ley de Newton en $\sum$ vemos que $\mathbf{F}$ actúa en dirección radial apuntando hacia el centro del disco (origen también de $\sum$ ').

De la ecuación (5) podemos observar que el primer término debe ser cero porque $\mathbf{r}$ y $\mathbf{F}$ son paralelos, lo cual anula el producto vectorial. En cuanto al segundo término, el producto $(\boldsymbol{\omega} \times \mathbf{r})$ representa la velocidad lineal de la partícula $v_{\tan } \widetilde{\theta}$, medida desde $\sum$. Pero el producto $\left(\boldsymbol{\omega} \times v_{\tan } \breve{\theta}\right)$ y el desplazamiento $d \mathbf{r}=r d \boldsymbol{\theta}$ son ortogonales, lo cual anula el producto escalar y en consecuencia el segundo término. Por otro lado, como hemos supuestow constante, el tercer término también debe ser cero.

Finalmente, resulta no sólo que $W_{\text {rot }}=0$, es decir que la diferencia entre $W$ y $W^{\prime}$ es cero, sino que ambos observadores están de acuerdo en el hecho de que la energía se conserva, a pesar de que midieron valores diferentes. Uno de ellos midió $K^{\prime}=0$, mientras que para el otro $K=\frac{m v_{\text {tan }}^{2}}{2}$.

Para un observador en $\sum$, la partícula describe una trayectoria circular como consecuencia directa de la acción dada por la fuerza de fricción. Esta fuerza cumple el rol de una fuerza centrípeta en ese marco. En todo momento, esta fuerza es perpendicular al desplazamiento, por lo cual no realiza trabajo y la energía permanece constante.

Para un observador en $\sum$ ' la partícula está en reposo, entonces no hay desplazamiento de la misma, y por lo tanto tampoco trabajo. La energía cinética se mantiene constante, con valor cero.

Un resultado similar se puede obtener si la partícula está en reposo en $\sum$.

\subsection{Problema 2}

Consideramos ahora el problema de una partícula moviéndose libremente en dirección radial, pero con una velocidad angular constante $\omega$ como una pelota moviındose dentro de un tubo rotante con movimiento circular uniforme como el mostrado en la Figura 2.

Visto desde $\sum$ ' la velocidad de la partícula se puede expresar como:

$$
v=v_{r a d}(t) \hat{r}
$$

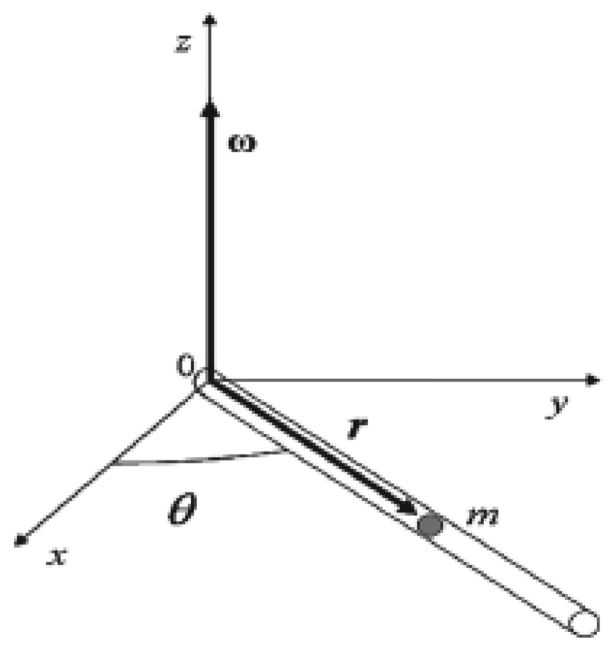

Figura 2: Partícula moviéndose por el interior de un tubo (Problema 2)

La fuerza de interacción que actúa sobre la partícula es sólo la fuerza normal realizada por el tubo $\mathbf{N}=0 \hat{r}+N \hat{\theta}$, expresada en coordenadas polares. Entonces las ecuaciones de Newton vistas por el observador en $\sum$ ' se pueden escribir como:

$$
m r \omega^{2}=m \ddot{r}
$$

para la dirección radial, y

$$
N-2 m \omega v_{r a d}=0
$$

para la tangencial.

$\mathrm{Al}$ integrar la ecuación radial llegamos a una solución de la forma:

$$
r(t)=r_{0} \cosh (\omega t)
$$

Donde $r_{0}$ es la distancia inicial al origen de coordenadas.

Integrando las ecuaciones (3) y (5) obtenemos:

$$
\begin{gathered}
W^{\prime}=\left.\frac{1}{2} m \omega^{2} r^{2}\right|_{r_{\text {inicial }}} ^{r_{\text {final }}} \\
W_{\text {rot }}=-\left.\frac{1}{2} m \omega^{2} r^{2}\right|_{r_{\text {inicial }}} ^{r_{\text {final }}}
\end{gathered}
$$

Esto se debe a que en el sistema rotante $\sum$ ', la única fuerza que realiza trabajo es la fuerza centrífuga.

Dado que, en principio, $W_{\text {rot }}$ es distinto de cero, la energía cinética en $\sum$ debe ser mayor que en 
$\sum$ '. Es decir, que un observador localizado en $\sum$ ' podría medir solo el trabajo realizado por la fuerza centrífuga. Mientras que otro observador situado en $\sum$ mediría la misma energía cinética más el trabajo realizado para preservar el sistema en movimiento rotacional.

De las ecuaciones anteriores obtenemos:

$$
W=\left.m \omega^{2} r^{2}\right|_{r_{\text {iniciak }}} ^{r_{\text {final }}}
$$

Para ambos observadores la energía no se conserva, y tampoco coinciden en el valor del trabajo realizado cuando la partícula va desde $r_{\text {inicial }}$ a $r_{\text {final }}$.

\subsection{Problema 3}

Supongamos un dispositivo similar al que se usó en el ejemplo anterior, pero ahora la partícula también se encuentra sometida a una fuerza radial dependiente de la posición, por lo tanto la fuerza neta aplicada es:

$$
\mathbf{F}=f(r) \hat{r}+N \hat{\theta}
$$

Por lo tanto, las ecuaciones de Newton vistas por el observador en $\sum$ ' pueden ser escritas como:

$$
f(r)+m r \omega^{2}=m \ddot{r}
$$

para la dirección radial, y

$$
N-2 m v_{\text {rad }} \omega=0
$$

para la dirección tangencial.

Algunos ejemplos de esta situación pueden ser: una partícula sometida a una tensión ejercida por un hilo que se acorta, obligando a la partícula a acercarse al eje de giro a velocidad $\mathbf{v}^{\prime}=v_{\text {rad }} \hat{r}$. Otra situación posible, la cual vamos a considerar, es una interacción elástica central con una constante elástica $k$. Bajo estas condiciones, la ecuación diferencial en el eje radial queda expresada del siguiente modo:

$$
\ddot{r}+\left(\omega^{2}-\frac{k}{m}\right) r=0
$$

Aquí, es necesario aclarar que la partícula puede presentar tres tipos de movimientos distintos de acuerdo al signo del coeficiente $\omega^{2}-\frac{k}{m}$. Cuando su valor es negativo, el movimiento radial puede ser descripto por una función hiperbólica. Por el contrario, si el valor del coeficiente es positivo, la partícula describe, radialmente, un movimiento oscilatorio. Finalmente, si el coeficiente es igual a cero, el vector $\mathbf{r}$ crece a velocidad constante.

Según el observador en $\sum$ ', el trabajo realizado sobre la partícula es:

$$
W^{\prime}=\left.\left(-\frac{1}{2} k r^{2}+\frac{1}{2} m \omega^{2} r^{2}\right)\right|_{r_{\text {iniciak }}} ^{r_{\text {final }}}
$$

Donde $W^{\prime}$ contiene las contribuciones de la fuerza elástica y de la fuerza centrífuga.

Por otro lado, integrando la ecuación (5) obtenemos que:

$$
W_{\text {rot }}=-\left.\frac{1}{2} m \omega^{2} r^{2}\right|_{r_{\text {iniciak }}} ^{r_{\text {final }}}
$$

Esta expresión es el resultado de dos contribuciones: el trabajo hecho por el momento de la normal tomando en cuenta que la normal es dependiente de la velocidad y el trabajo de la fuerza centrífuga.

Entonces, de la ecuación (3) el trabajo visto por el observador en $\sum$ es:

$$
W=\left.\left(-\frac{1}{2} k r^{2}+m \omega^{2} r^{2}\right)\right|_{r_{\text {iniciak }}} ^{r_{\text {final }}}
$$

$W$ también tiene dos contribuciones: una asociada al potencial elástico y otra que proviene del trabajo de la normal, que es una fuerza no conservativa que realiza trabajo y por lo tanto la energía no se conserva. De esta forma, ambos observadores $\sum$ y $\sum$ ' concuerdan en la no conservación de la energía mecánica, aunque lo atribuyen a diferentes mecanismos. Para el observador rotante, es debido al trabajo de la fuerza ficticia. Para el observador inercial hay un agente externo que obliga al tubo a girar con velocidad angular constante.

\subsection{Problema 4}

Extendemos el caso anterior considerando el caso de un sistema de dos partículas con diferente masa situadas en un riel rotante sin fricción y que interactúan elásticamente (supondremos que la longitud relajada del resorte es nula, ver Figura 3). Las fuerzas que actúan sobre cada partícula en el plano de movimiento son la normal y la fuerza elástica. 


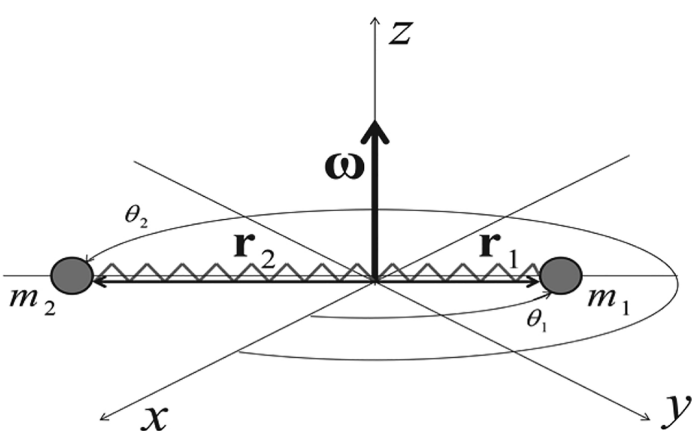

Figura 3: Partículas sometidas a fuerzas elásticas en sistema rotante (Problema 4)

Con el propósito de estudiar el problema rotacional, situamos el centro de masa del sistema (con velocidad cero) en el centro de rotación y consideramos coordenadas polares.

Cabe aclarar, que de la misma forma que en el problema 3, las masas pueden adquirir movimientos distintos según las condiciones iniciales y de configuración del sistema. Sin embargo, para cualquiera de las situaciones posibles, el resultado del análisis del trabajo y la energía es el mismo.

Las fuerzas netas que actúan sobre cada partícula son:

$$
\begin{aligned}
& \mathbf{F}_{1}=-k\left(r_{1}+r_{2}\right) \hat{r}+N_{1} \hat{\theta} \\
& \mathbf{F}_{2}=-k\left(r_{2}+r_{1}\right) \hat{r}+N_{2} \hat{\theta}
\end{aligned}
$$

Calculando el trabajo medido por un observador en $\sum$ ' obtenemos:

$$
\begin{aligned}
& W^{\prime}=\left(-\frac{1}{2} k\left(r_{1}+r_{2}\right)^{2}\right. \\
& \left.+\frac{1}{2} \omega^{2}\left(m_{1} r_{1}^{2}+m_{2} r_{2}^{2}\right)\right)\left.\right|_{\left(r_{1 i}, r_{2 i}\right)} ^{\left(r_{1 f}, r_{2 f}\right)}
\end{aligned}
$$

y $\left(r_{1 i}, r_{2 i}\right)$ representan las posiciones final/inicial de las partículas 1 y 2 .

Integrando la ecuación (5), considerando que ahora el sistema está formado por dos partículas, obtenemos que el trabajo de rotación es:

$$
W_{\text {rot }}=-\left.\frac{1}{2} \omega^{2}\left(m_{1} r_{1}^{2}+m_{2} r_{2}^{2}\right)\right|_{\left(r_{1 i}, r_{2 i}\right)} ^{\left(r_{1 f}, r_{2 f}\right)}
$$

Análogamente, al problema anterior el trabajo calculado por el observador inercial es ahora:

$$
\begin{aligned}
& W=\left(-\frac{1}{2} k\left(r_{1}+r_{2}\right)^{2}\right. \\
& \left.+\omega^{2}\left(m_{1} r_{1}^{2}+m_{2} r_{2}^{2}\right)\right)\left.\right|_{\left(r_{1 i}, r_{2 i}\right)} ^{\left(r_{1 f}, r_{2 f}\right)}
\end{aligned}
$$

Las conclusiones generales son las mismas del problema anterior. La extensión al caso de más partículas, aunque requiere más trabajo algebraico, no aportaría a la discusión conceptual.

\section{Conclusiones}

El resultado de que el trabajo hecho por la fuerza neta que actúa sobre una partícula corresponde a su cambio de energía cinética es un concepto central de la Física. En la mayoría de los libros de texto está usualmente declarado en un dado sistema de referencia y raramente relacionado al cambio del sistema de referencia en movimiento traslacional ([1], [2]). Por otro lado, el hecho de que existen ciertos problemas que involucran sistemas rotantes, nos motiva a extender esos resultados al caso de movimiento relativo rotacional.

Mostramos cómo presentar explícitamente la diferencia en la energía cinética que es vista por un observador inercial y no inercial (rotacional) de una manera intuitiva y concreta. Realizamos los cálculos para un sistema de una única partícula, mostrando con un problema cómo sería posible extenderlo a sistemas de más partículas. Por lo que sabemos, no hemos encontrado una discusión de este tema junto con una propuesta de actividades a desarrollar en cursos básicos de física universitaria que apunten a lograr una presentación sencilla pero manteniendo un profundo alcance conceptual. En [11] se propone un abordaje en esta dirección, aunque con un enfoque menos didáctico y sin una propuesta de problemas para introducir gradualmente el tema en sistemas de una y varias partículas.

Aquí (y en una versión preliminar en [10]) mostramos una manera general, y sin invocar conceptos avanzados de la mecánica teórica [7], que el trabajo hecho por la fuerza centrífuga, la rotación no uniforme y el torque de fuerzas de interacción son los responsables del desacuerdo sobre la conservación de la energía entre dos observadores en rotación relativa. El trabajo hecho por la fuerza de Coriolis es cero, cualquiera sea el movimiento de la partícula. Ilustramos estos resultados con varios problemas típicos que ayudan a comprender el comportamiento 
diferente visto por dos observadores. Para que la discusión resulte más clara, nos hemos restringido a situaciones con rotación uniforme. Además, la secuencia en que se presenta el abordaje del problema, facilitaría el alcance de nuestra meta central en cuanto a la enseñanza de este tema. Según enunciamos al comienzo, propendemos a que los alumnos logren revisar críticamente lo aprendido con anterioridad, apreciando los límites de validez de la descripción de un problema en el caso particular del Teorema de la Energía Cinética.

\section{Apéndice}

Una partícula de masa $m$ tendrá posición $\mathbf{r}$ en $\sum$ y r ' en $\sum$ '. Mientras el origen de coordenadas coincida, la posición de las partículas será la misma en ambos sistemas pero estarán expresadas en coordenadas diferentes: $\mathbf{r}^{\prime}=\mathbf{M r}$, siendo $\mathbf{M}$ la matriz que relaciona los vectores en $\sum$ y en $\sum$ '.

Para cualquier desplazamiento $d \boldsymbol{r}$ en $\sum$ habrá una rotación adicional $d \boldsymbol{\theta} \times \mathbf{r}$ en $\sum$ ', donde $d \boldsymbol{\theta}$ es el cambio angular entre ambos sistemas durante el movimiento. De esta forma, obtenemos [8]:

$$
d \mathbf{r}^{\prime}=d \mathbf{r}-d \boldsymbol{\theta} \times \mathbf{r}
$$

el cual corresponde al desplazamiento en el sistema $\sum$ '. Debido a que consideramos una aproximación no relativista, los intervalos de tiempo y las masas son invariantes bajo cambios del sistema de referencia, entonces [8]:

$$
\mathbf{v}^{\prime}=\mathbf{v}-\omega \times \mathbf{r}
$$

$\mathrm{y}$

$$
\begin{aligned}
& d \mathbf{v}^{\prime}=d \mathbf{v}-2 d \boldsymbol{\theta} \times \mathbf{v}^{\prime}-\boldsymbol{\omega} \times\left(d \boldsymbol{\theta} \times \mathbf{r}^{\prime}\right) \\
& -d \boldsymbol{\omega} \times \mathbf{r}^{\prime}
\end{aligned}
$$

Supongamos que hay una fuerza neta $\boldsymbol{F}$ actuando sobre la partícula, luego un observador en $\sum$ ' tiene que incluir las llamadas fuerzas inerciales (Coriolis, fuerza centrífuga y una asociada a la aceleración angular) para conservar la segunda ley de Newton:

$$
\begin{aligned}
& m \frac{d \mathbf{v}^{\prime}}{d t}=\mathbf{F}-m\left[\frac{d \boldsymbol{\omega}}{d t} \times \mathbf{r}^{\prime}+2 \boldsymbol{\omega} \times \mathbf{v}^{\prime}\right. \\
& \left.+\boldsymbol{\omega} \times \boldsymbol{\omega} \times \mathbf{r}^{\prime}\right]
\end{aligned}
$$

Aplicando la definición usual de trabajo en un marco rotacional, el TTE todavía es válido y es se expresa como: $[1,10]$.

$$
\begin{aligned}
& m d \mathbf{v}^{\prime} \cdot \mathbf{v}^{\prime}=\left[\mathbf{F}-m \frac{d \boldsymbol{\omega}}{d t} \times \mathbf{r}^{\prime}-2 m \boldsymbol{\omega} \times \mathbf{v}^{\prime}\right. \\
& \left.-m\left(\boldsymbol{\omega} \times \boldsymbol{\omega} \times \mathbf{r}^{\prime}\right)\right] \cdot d \mathbf{r}^{\prime}
\end{aligned}
$$

Y en forma abreviada: $d K^{\prime}=d W^{\prime}$.

Es posible relacionar las cantidades medidas en ambos sistemas, para poder ver que el trabajo medido en $\sum$ ' es igual al trabajo hecho por las fuerzas de interacción (como las medidas en $\sum$ ) más un término debido a las fuerzas inerciales:

$$
d W^{\prime}=d W+d W_{\text {rot }}
$$

Consideramos el trabajo visto por un observador en $\sum^{\prime}$

$$
\begin{aligned}
& d W^{\prime}=\mathbf{F} \cdot d \mathbf{r}^{\prime}-2 m\left(\boldsymbol{\omega} \times \mathbf{v}^{\prime}\right) \cdot d \mathbf{r}^{\prime} \\
& -m\left(\boldsymbol{\omega} \times \boldsymbol{\omega} \times \mathbf{r}^{\prime}\right) \cdot d \mathbf{r}^{\prime}- \\
& m\left(\frac{d \boldsymbol{\omega}}{d t} \times \mathbf{r}^{\prime}\right) \cdot d \mathbf{r}^{\prime}
\end{aligned}
$$

Como el producto $\boldsymbol{\omega} \times \mathbf{v}^{\prime}$ es perpendicular al desplazamiento $d \mathbf{r}^{\prime}$,el producto escalar anula el segundo término. Físicamente, esto implica que la fuerza de Coriolis no realiza trabajo en el sistema rotacional porque actúa en una dirección que siempre es perpendicular al desplazamiento de la partícula. Sería posible hacer una analogía entre la fuerza de Coriolis y la de Lorentz: en ambos casos la fuerza es el producto vectorialentre la velocidad y un vector externo (la velocidad angular $\boldsymbol{\omega}$ o el vector del campo magnético $\boldsymbol{B})$, de manera que la fuerza es ortogonal a la trayectoria siempre y por lo tanto no realiza trabajo alguno.

Realizando algo de álgebra obtenemos:

$$
\begin{aligned}
& d W_{\text {rot }}=-d \boldsymbol{\theta} \cdot(\mathbf{r} \times \mathbf{F})-m(\boldsymbol{\omega} \times \boldsymbol{\omega} \times \mathbf{r}) \cdot d \mathbf{r} \\
& -m\left(\mathbf{r} \times \frac{d \mathbf{r}}{d t}\right) \cdot d \boldsymbol{\omega}
\end{aligned}
$$

donde

$$
d W=\mathbf{F} \cdot d \mathbf{r}
$$

representa el trabajo en el sistema $\sum$. 


\section{Referencias}

[1] M. Camarca, A. Bonanno and P. Sapia, Eur. J. Phys. 28, 1181 (2007).

[2] Rodolfo A. Diaz, William J. Herrera and Diego A. Manjarres, Am. J. Phys 77, 270 (2008).

[3] R.A. Serway and J.W. Jewett, Physics for Scientists and Engineers (Saunders Golden Sunburst Series, Fort Worth, 2007).

[4] R. Feynman, R. Leighton and M. Sands, The Feynman Lectures on Physics (Addison-Wesley, Reading, 1964).

[5] A.B. Arons, Am. J. Phys. 67, 1063 (1999).

[6] B.A. Sherwood, Am. J. Phys. 51, 597 (1983).

[7] L. Landau and M. Lifshiftz, Mechanics (Pergamon, London, 1959).

[8] H. Goldstein, C. Poole and J. Safko, Classical Mechanics (Addison Wesley, Reading, 2002), 3rd ed.

[9] M. Stone Wiske, La Enseñanza para la Comprensión. Vinculación entre la Investigación y la Práctica (Paidos, Buenos Aires, 1999).

[10] D.M. Fernández, M.F. Carusela and C.D. El Hasi, arXiv:1101.0157v1 (2010).

[11] D.A. Manjarres, W.J. Herrera and R.A. Diaz, Am. J. Phys. 81, 597 (2013). 